OPEN ACCESS

Edited by:

Ying-Chun Xu,

Peking Union Medical College

Hospital (CAMS), China

Reviewed by:

Ping Zhan

Jiangxi Provincial People's Hospital,

China

Changbin Chen,

Institut Pasteur of Shanghai (CAS),

China

${ }^{*}$ Correspondence:

Wenjuan Wu

wwj1210@126.com

Heping Xu

xmsunxhp@163.com

Lingbing Zeng

lingbing_zeng@163.com

tThese authors have contributed equally to this work

Specialty section: This article was submitted to

Antimicrobials, Resistance and Chemotherapy,

a section of the journal

Frontiers in Microbiology

Received: 19 December 2020 Accepted: 26 January 2021

Published: 04 March 2021

Citation:

Guo J, Zhang M, Qiao D, Shen H, Wang L, Wang D, Li L, Liu Y, Lu H, Wang C, Ding H, Zhou S, Zhou W,

Wei Y, Zhang $H$, Xi W, Zheng Y, Wang $Y$, Tang $R$, Zeng $L, X u H$ and Wu W (2021) Prevalence

and Antifungal Susceptibility of Candida parapsilosis Species Complex in Eastern China: A 15-Year

Retrospective Study by ECIFIG.

Front. Microbiol. 12:644000. doi: 10.3389/fmicb.2021.644000

\section{Prevalence and Antifungal Susceptibility of Candida parapsilosis Species Complex in Eastern China: A 15-Year Retrospective Study by ECIFIG}

Jian Guo ${ }^{1 \dagger}$, Min Zhang ${ }^{1+}$, Dan Qiao ${ }^{2 \dagger}$, Hui Shen ${ }^{1}$, Lili Wang ${ }^{1}$, Dongjiang Wang ${ }^{1}$, Li Li ${ }^{2}$, Yun Liu ${ }^{3}$, Huaiwei Lu ${ }^{4}$, Chun Wang ${ }^{5}$, Hui Ding ${ }^{6}$, Shuping Zhou ${ }^{7}$, Wanqing Zhou ${ }^{8}$, Yingjue Wei ${ }^{9}$, Haomin Zhang ${ }^{9}$, Wei Xi ${ }^{9}$, Yi Zheng ${ }^{10}$, Yueling Wang ${ }^{11}$, Rong Tang ${ }^{12}$, Lingbing Zeng ${ }^{13 *}$, Heping $\mathrm{X}{ }^{14 *}$ and Wenjuan $\mathrm{Wu}^{1 *}$

\footnotetext{
'Department of Laboratory Medicine, Shanghai East Hospital, Tongii University School of Medicine, Shanghai, China, ${ }^{2}$ Department of Laboratory Medicine, Ruijin Hospital, Shanghai Jiao Tong University School of Medicine, Shanghai, China, ${ }^{3}$ Department of Laboratory Medicine, Changhai Hospital, Naval Medical University (Second Military Medical University), Shanghai, China, ${ }^{4}$ Department of Laboratory Medicine, The First Affiliated Hospital of USTC, Hefei, China, ${ }^{5}$ Department of Laboratory Medicine, Children's Hospital, Shanghai Jiao Tong University School of Medicine, Shanghai, China,

${ }^{6}$ Department of Laboratory Medicine, Lishui Municipal Central Hospital, Lishui, China, ${ }^{7}$ Department of Laboratory Medicine, Jiangxi Provincial Children's Hospital, Nanchang, China, ${ }^{8}$ Department of Laboratory Medicine, Nanjing Drum Tower Hospital, The Affiliated Hospital of Nanjing University Medical School, Nanjing University, Nanjing, China, ${ }^{9}$ Department of Laboratory Medicine, Renji Hospital, Shanghai Jiao Tong University School of Medicine, Shanghai, China, ${ }^{10}$ Department of Clinical Laboratory, The Second Affiliated Hospital of Soochow University, Suzhou, China, ${ }^{11}$ Department of Clinical Laboratory, Shandong Provincial Hospital Affiliated to Shandong First Medical University, Jinan, China, ${ }^{12}$ Department of Laboratory Medicine, Shanghai General Hospital, Shanghai Jiao Tong University School of Medicine, Shanghai, China, ${ }^{13}$ Department of Laboratory Medicine, The First Affiliated Hospital of Nanchang University, Nanchang, China, ${ }^{14}$ Department of Laboratory Medicine, The First Affiliated Hospital of Xiamen University, Xiamen, China
}

Candida parapsilosis complex is one of the most common non-albicans Candida species that cause candidemia, especially invasive candidiasis. The purpose of this study was to evaluate the antifungal susceptibilities of both colonized and invasive clinical C. parapsilosis complex isolates to 10 drugs: amphotericin (AMB), anidulafungin (AFG), caspofungin (CAS), micafungin (MFG), fluconazole (FLZ), voriconazole (VRZ), itraconazole (ITZ), posaconazole (POZ), 5-flucytosine (FCY), and isaconazole (ISA). In total, 884 C. parapsilosis species complex isolates were gathered between January 2005 and December 2020. C. parapsilosis, Candida metapsilosis, and Candida orthopsilosis accounted for $86.3,8.1$, and $5.5 \%$ of the cryptic species, respectively. The resistance/non-wild-type rate of bloodstream $C$. parapsilosis to the drugs was $3.5 \%$, of C. metapsilosis to AFG and CAS was 7.7\%, and of C. orthopsilosis to FLZ and VRZ was $15 \%$ and to CAS, MFG, and POZ was 5\%. The geometric mean (GM) minimum inhibitory concentrations (MICs) of non-bloodstream C. parapsilosis for CAS (0.555 mg/L), MFG (0.853 mg/L), FLZ (0.816 mg/L), VRZ (0.017 mg/L), ITZ $(0.076 \mathrm{mg} / \mathrm{L})$, and $\mathrm{POZ}(0.042 \mathrm{mg} / \mathrm{L})$ were significantly higher than those of bloodstream C. parapsilosis, for which the GM MICs were $0.464,0.745,0.704,0.015,0.061$ and $0.033 \mathrm{mg} / \mathrm{L}$, respectively $(P<0.05)$. The $\mathrm{MIC}$ distribution of the bloodstream C. parapsilosis strains collected from 2019 to 2020 for VRZ, POZ, and ITZ were 
$0.018,0.040$, and $0.073 \mathrm{mg} / \mathrm{L}$, significantly higher than those from 2005 to 2018, which were $0.013,0.028$, and $0.052 \mathrm{mg} / \mathrm{L}(P<0.05)$. Additionally, MIC distributions of C. parapsilosis with $\mathrm{FLZ}$ and the distributions of $C$. orthopsilosis with ITZ and POZ might be higher than those in Clinical and Laboratory Standards Institute studies. Furthermore, a total of 143 C. parapsilosis complex isolates showed great susceptibility to ISA. Overall, antifungal treatment of the non-bloodstream C. parapsilosis complex isolates should be managed and improved. The clinicians are suggested to pay more attention on azoles usage for the $C$. parapsilosis complex isolates. In addition, establishing the epidemiological cutoff values (ECVs) for azoles used in Eastern China may offer better guidance for clinical treatments. Although ISA acts on the same target as other azoles, it may be used as an alternative therapy for cases caused by FLZ- or VRZ-resistant C. parapsilosis complex strains.

Keywords: antifungal susceptibility, Candida parapsilosis, Candida metapsilosis, Candida orthopsilosis, China

\section{INTRODUCTION}

Candida albicans and emerging non-albicans Candida species can result in superficial infections of the oral and vaginal mucosa, as well as invasive candidiasis, such as bloodstream infections and deep-tissue infections. These invasive infections are associated with high mortality of about 70\% (Pappas et al., 2018). C. albicans is the most common and aggressive species causing Candida infections around the world. However, over the past few decades, non-albicans Candida species such as Candida glabrata, Candida parapsilosis, and Candida tropicalis have also become health concern (Chow et al., 2008; Silva et al., 2012; Guinea, 2014; Guo et al., 2017). Among them, C. parapsilosis is well known for its threat to the patients undergoing invasive medical interventions, as it is considered to be one of the leading causes of catheter-related infections and can form enhanced biofilms on central venous catheters (CVCs) and other medical implants (Trofa et al., 2008; Pfaller et al., 2014; Strollo et al., 2016; Tóth et al., 2019). C. parapsilosis is also the second or third most commonly isolated Candida species in the intensive care units (ICUs) (Magobo et al., 2017; Asadzadeh et al., 2019). In contrast to C. albicans, horizontal transmission is another characteristic of $C$. parapsilosis, which allows the species to spread through contaminated medical equipment and medical staff in the clinic, leading to crossover infections between patients (García San Miguel et al., 2004; Kuhn et al., 2004; Vaz et al., 2011).

Candida parapsilosis, C. metapsilosis, and C. orthopsilosis are three species of the C. parapsilosis species complex (Tavanti et al., 2005). The prevalence of $C$. parapsilosis is the highest among the cryptic species. A 6-year multicenter study in Iran reported that the proportions of C. orthopsilosis and C. metapsilosis were quite small, comprising 5.3 and $0.17 \%$ of all C. parapsilosis species complex isolates (Arastehfar et al., 2019). While in Argentina and India, C. orthopsilosis may account for a higher proportion, reaching $40 \%$ of the cryptic species (Maria et al., 2018; Vigezzi et al., 2019). C. parapsilosis complex isolates were found susceptible to most of the antifungal agents (Modiri et al., 2019; Vigezzi et al., 2019). The multicenter studies in China by the China Hospital Invasive Fungal Surveillance Net
(CHIF-NET) performed in 2015, 2018, and 2020 all reported low resistance/non-wild-type (NWT) rate of C. parapsilosis complex isolates to azoles $(<6 \%)$ (Xiao et al., 2015, 2018, 2020). The multicenter study in Iran also reported four NWT C. orthopsilosis isolates for itraconazole (ITZ) (Arastehfar et al., 2019). Yet, the work in India reported high resistance to fluconazole (FLZ) of C. parapsilosis (Maria et al., 2018). However, all of the studies lasted only 2-6 years, lacking long-term studies.

Additionally, the difference in antifungal susceptibilities between the three cryptic species were described. Vigezzi et al. (2019) reported lower minimal inhibitory concentrations (MICs) with C. parapsilosis than C. orthopsilosis for ITZ and higher MIC values for echinocandins $(P<0.01)$. Similarly, GilAlonso et al. (2019) reported that C. metapsilosis was the most susceptible species to echinocandins, followed by C. orthopsilosis and C. parapsilosis. However, the number of strains used in these studies were quite limited so that these results may not able to uncover the difference clearly.

Furthermore, the Clinical and Laboratory Standards Institute (CLSI) updated its document M60-Ed2 in June 2020, illustrating that the clinical breakpoints can only be used for C. parapsilosis; otherwise, the epidemiological cutoff values (ECVs) recommended by M59-Ed3 should be applied for C. metapsilosis and C. orthopsilosis. No large-scale study, except the programs associated with CLSI, has been performed since then.

Notably, only the C. parapsilosis complex isolates from invasive candidiasis have been studied for their antifungal susceptibilities so far. C. parapsilosis complex are opportunistic pathogens that may transition from colonization to invasion; therefore, we believe that it is also worth studying colonized isolates. We launched the Eastern China Invasive Fungi Infection Group (ECIFIG), a multicenter institute, in 2019 in Shanghai to supervise the Candida strains isolated from both colonization and invasion sites and to improve rapid fungal diagnosis, therapeutic drug monitoring, and clinical intervention teams.

Therefore, this 15-year multicenter study collected a total of 884 colonized and invasive C. parapsilosis complex isolates in eastern China, with the aim of investigating their 
epidemiological characteristics and antifungal susceptibility distributions systematically with nine common antifungal drugs as well as isavuconazole (ISA), a drug whose ECV was first reported in CLSI M59-Ed3 for C. duobushaemulonii only, applying the clinical breakpoints and ECVs updated in CLSI M60-Ed2 and M59-Ed3.

\section{MATERIALS AND METHODS}

\section{Strains}

For this retrospective study, a total of 884 C. parapsilosis complex clinical isolates (763 C. parapsilosis, 49 C. orthopsilosis, and 72 C. metapsilosis) were collected from 835 patients with more than one episode of candidiasis. The isolates were gathered from different tertiary hospitals of ECIFIG between 2005 and 2020. Among the seven provinces in Eastern China, the majority of the isolates were from Shanghai $(n=520)$, Jiangxi $(n=240)$, and Fujian $(n=64)$ (Table 1). For each isolate, the collected information included age and gender of the patient, date of sample collection, specimen type, body site of isolation, and the ward location of the patient at the time of sample collection. Strains were isolated from clinical samples of patients with bloodstream and non-bloodstream fungal infections.

\section{Strains Identification}

All isolates were first identified by biochemical methods and then confirmed by matrix-assisted laser desorption ionization-time of flight mass spectrometry (MALDITOF) (Autof ms1000, Autobio). Sequencing of the internal transcribed spacer (ITS) ribosomal DNA (rDNA) (ITS1/ITS4) region was performed for definitive species identification.

TABLE 1 | Number of C. parapsilosis complex isolates collected from different hospitals.

\begin{tabular}{|c|c|c|c|c|}
\hline \multicolumn{2}{|r|}{ Hospitals } & \multicolumn{3}{|c|}{ No. of isolates (\%) } \\
\hline & & C. parapsilosis $(n=763)$ & C. metapsilosis $(n=72)$ & C. orthopsilosis $(n=49)$ \\
\hline \multirow[t]{22}{*}{ Shanghai } & East Hospital & $185(24.2)$ & $2(2.8)$ & $4(8.2)$ \\
\hline & Ruijin Hospital & $35(4.6)$ & & $6(12.2)$ \\
\hline & Shanghai Children's Hospital & $34(4.5)$ & $1(1.4)$ & $1(2)$ \\
\hline & Changhai Hospital & $26(3.4)$ & $1(1.4)$ & $11(22.4)$ \\
\hline & Changzheng Hospital & $26(3.4)$ & & \\
\hline & Shanghai General Hospital & $26(3.4)$ & $1(1.4)$ & \\
\hline & Shanghai Tenth People's Hospital & $23(3)$ & $3(4.2)$ & \\
\hline & Eye and Ent Hospital of Fudan University & $19(2.5)$ & $5(6.9)$ & $2(4.1)$ \\
\hline & East Brach of Renji Hospital & $14(1.8)$ & $2(2.8)$ & $1(2)$ \\
\hline & Shanghai Public Health Clinical Center & $13(1.7)$ & & \\
\hline & West Brach of Renji Hospital & $12(1.6)$ & $3(4.2)$ & \\
\hline & Zhongshan Hospital & $11(1.4)$ & & \\
\hline & Children's Hospital of Fudan University & $9(1.2)$ & & $1(2)$ \\
\hline & Huadong Hospital & $9(1.2)$ & & \\
\hline & Shanghai Children's Medical Center & $8(1)$ & & \\
\hline & South Brach of Renji Hospital & $6(0.8)$ & & \\
\hline & Huashan Hospital & $5(0.7)$ & & \\
\hline & Shanghai Eastern Hepatobiliary Surgery Hospital & $4(0.5)$ & & \\
\hline & Shanghai Chest Hospital & $4(0.5)$ & & \\
\hline & Shanghai Cancer Center & $4(0.5)$ & & \\
\hline & Shanghai Pudong New Area Gongli Hospital & $3(0.4)$ & & \\
\hline & Total & $476(62.4)$ & $18(25)$ & $26(53.1)$ \\
\hline \multirow[t]{4}{*}{ Jiangxi } & The First Affiliated Hospital of Nanchang University & $168(22)$ & $41(56.9)$ & $21(42.9)$ \\
\hline & Jiangxi Provincial People’s Hospital & $9(1.2)$ & & \\
\hline & Jiangxi Provincial Children’s Hospital & $1(0.1)$ & & \\
\hline & Total & $178(23.3)$ & & \\
\hline Fujian & The First Affiliated Hospital of Xiamen University & $51(6.7)$ & $11(15.3)$ & $2(4.1)$ \\
\hline Zhejiang & Zhejiang Lishui Central Hospital & $19(2.5)$ & & \\
\hline Anhui & Anhui Provincial Hospital & $18(2.4)$ & $2(2.8)$ & \\
\hline \multirow[t]{3}{*}{ Jiangsu } & The First Affiliated Hospital of Soochow University & $9(1.2)$ & & \\
\hline & Nanjing Drum Tower Hospital & $8(1)$ & & \\
\hline & Total & $17(2.2)$ & & \\
\hline Shandong & Shandong Provincial Hospital & $4(0.5)$ & & \\
\hline
\end{tabular}




\section{Criteria for Study Inclusion}

We collected all C. parapsilosis complex isolates recovered from the blood and non-blood of patients and included them in this study. Isolates from bronchoalveolar lavage fluid (BALF), CVC tips, and the gastrointestinal tracts of patients with invasive infections were tested. Isolates from sputum, urine, genital tract, and other places considered to be colonizers were also collected as non-blood strains. Isolates of the same species with the same susceptibility or resistance biotype profile from the same site of the same patient at different times were considered duplicates and were excluded.

\section{Criteria for Grouping}

Since ECIFIG was launched in 2019 and started to collect all the strains isolated clinically, we divide the bloodstream C. parapsilosis isolates into two groups-2005-2018 and 2019-2020-to study the difference for MIC distributions between the two groups.

\section{Antifungal Susceptibility Testing}

The in vitro susceptibility of the 741 C. parapsilosis complex strains to nine antifungal drugs, amphotericin $\mathrm{B}$ (AMB, $0.12-8 \mathrm{mg} / \mathrm{L}$ ), anidulafungin (AFG, $0.015-8 \mathrm{mg} / \mathrm{L}$ ), caspofungin (CAS, $0.008-8 \mathrm{mg} / \mathrm{L}$ ), micafungin (MFG, $0.008-8 \mathrm{mg} / \mathrm{L}$ ), FLZ (0.12-256 mg/L), VRZ (0.008-8 mg/L), ITZ (0.015-16 mg/L), posaconazole (POZ, $0.008-8 \mathrm{mg} / \mathrm{L}$ ), and 5-flucytosine (FCY, $0.06-64 \mathrm{mg} / \mathrm{L}$ ) were determined by the Sensititre YeastOne ${ }^{\mathrm{TM}}$ YO10 methodology (Thermo Fisher Scientific, Waltham, MA, United States) following the manufacturer's instructions. The in vitro antifungal susceptibility tests of the other 143 C. parapsilosis complex strains collected in 2020 were conducted using the Sensititre YeastOne $\mathrm{TM}^{\mathrm{TM}} \mathrm{CMC1JHY}$ methodology (Thermo Fisher Scientific), which is a new plate for research only, in which $\mathrm{AMB}$ is replaced with isavuconazole (ISA, $0.015-16 \mathrm{mg} / \mathrm{L}$ ), and the concentration of CAS is changed from $0.008-8 \mathrm{mg} / \mathrm{L}$ to $0.06-4 \mathrm{mg} / \mathrm{L}$. For these 143 strains, the in vitro susceptibilities to AMB were determined by the AMB microbroth dilution kit (BIO-KONT ${ }^{\circledR}$, Wenzhou, China), which is also for scientific research only, with the drug concentrations ranging from 0.125 to $8 \mathrm{mg} / \mathrm{L}$. C. parapsilosis (ATCC 22019) and C. krusei (ATCC 6258) standard strains were used as quality controls. After being incubated at $35^{\circ} \mathrm{C}$ for $24 \mathrm{~h}$, the MIC endpoints were determined following the manufacturer's instructions. For Sensititre YeastOne ${ }^{\mathrm{TM}}$ YO10 and CMC1JHY (Thermo Fisher Scientific), MIC was defined as the lowest drug concentration at which the color in the well changed from red to blue. For the AMB microbroth dilution kit (BIO-KONT ${ }^{\oplus}$ ), MIC was defined as the lowest concentration at which there was $100 \%$ growth inhibition. $\mathrm{MIC}_{50}$ and $\mathrm{MIC}_{90}$ were defined as the MICs required to inhibit the growth of 50 and $90 \%$ of the organisms, respectively. Data were interpreted based on the clinical breakpoints recommended by the CLSI M60-Ed2. Accordingly, the ECVs recommended by CLSI M59-Ed3 were applied if further species determination identified one of the cryptic species within the complex.

\section{Ethical Approval}

This retrospective study was approved by the ethics committee of Shanghai East Hospital, Tongji University School of Medicine. The need for informed consents was waived by the Clinical Research Ethics Committee [(2017) Pre-examination No. 026].

\section{Statistical Analysis}

The results were initially evaluated to assess if the data exhibited asymmetry and/or high variances using the MannWhitney test to compare the MIC distributions between non-bloodstream and bloodstream isolates as well as to compare the MIC distributions of bloodstream C. parapsilosis isolates between 2015-2018 and 2019-2020. The KruskalWallis tests were used to compare the MIC distributions between the three cryptic species. $P<0.05$ indicated statistical significance. Statistical analyses were performed using IBM SPSS for Windows v22.0 (IBM, Armonk, NY, United States). Antifungal susceptibility results that were " $\leq$ the lowest concentration" were defaulted as "= the lowest concentration" for statistical analysis. Figures were made using GraphPad Prism v8.0 (GraphPad Software, Inc., San Diego, CA, United States).

\section{RESULTS}

\section{Epidemiological Characteristics}

The number of C. parapsilosis strains isolated were $334(43.8 \%)$ from 2019 to 2020 and 429 (56.2\%) from 2005 to 2018; C. metapsilosis 30 (41.7\%) from 2019 to 2020 and 42 (58.3\%) from 2005 to 2018; and C. orthopsilosis 15 (30.6\%) from 2019 to 2020 and 34 (69.4\%) from 2005 to 2018 (Table 2). Regarding patients with isolated strains, the age distributions among the three species were similar: $54.46 \pm 23.29$ years for C. parapsilosis, $52.38 \pm 19$ years for C. metapsilosis, and $51.21 \pm 23.98$ years for C. orthopsilosis. In addition, strains were nearly two times more frequently isolated from male than female patients. The variety of sample sources of $C$. parapsilosis was higher than that of C. orthopsilosis and C. metapsilosis. C. parapsilosis strains were mainly isolated from blood (37.1\%), followed by sputum $(11.5 \%)$, urine $(10.4 \%)$, stool $(7.2 \%)$, and CVC (6.6\%). The proportion of C. orthopsilosis strains isolated from blood was highest $(40.8 \%)$, followed by stool $(14.3 \%)$, vagina $(10.2 \%)$, sputum $(8.2 \%)$, urine $(8.2 \%)$, and CVC $(4.1 \%)$. The percentage of strains isolated from blood was the lowest for C. metapsilosis among the three species (18.1\%), followed by sputum (18.1\%), stool (15.3\%), and vagina (11.1\%). The proportion of C. metapsilosis isolated from CVC was also the lowest among the three species (2.8\%). The top 10 ward where C. parapsilosis complexes were isolated were ICU (12.4\%), Oncology (11.5\%), Emergency Medicine (8.9\%), Gynecology (7.8\%), Gastroenterology (7.0\%), Respiratory Medicine (6.9\%), Nephrology (5.9\%), Hepatobiliary and Pancreatic Surgery (5.3\%), Neonatology (4.0\%), and Otorhinolaryngology (3.6\%). 
TABLE 2 | Epidemiological characteristics of the C. parapsilosis complex isolates.

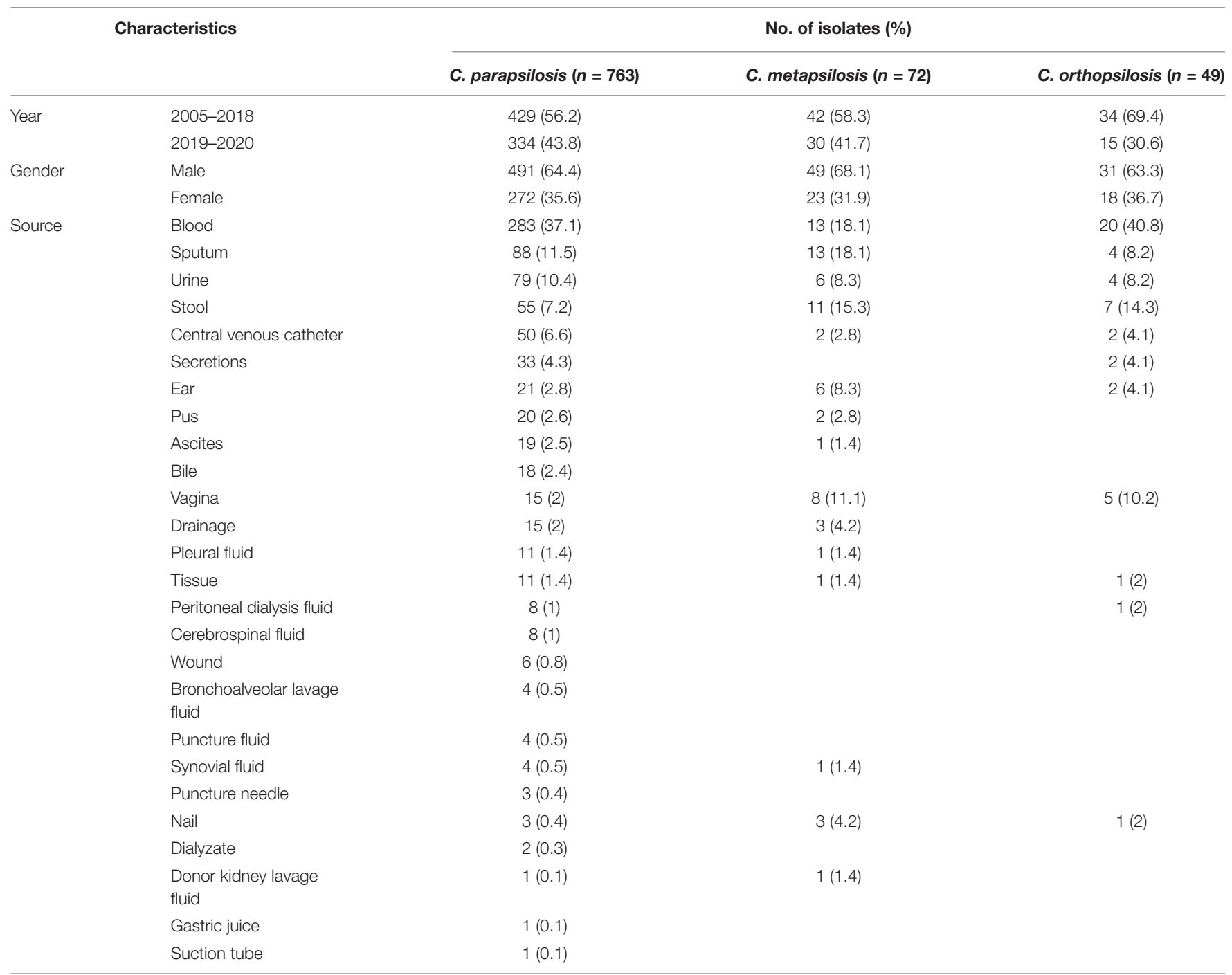

\section{Antifungal Susceptibility Results Resistance/NWT Rates of the Candida parapsilosis Complex Isolates}

Among the 283 bloodstream C. parapsilosis, there were $3.2 \%$ SDD and $3.5 \% \mathrm{R}$ to FLZ, $1.4 \% \mathrm{I}$ and $2.5 \% \mathrm{R}$ to $\mathrm{VRZ}, 0.4 \% \mathrm{I}$ to MFG, $0.4 \%$ NWT to ITZ, $1.4 \%$ NWT to POZ, and $1.1 \%$ NWT to AMB (Supplementary Table 2). Among the 13 bloodstream C. metapsilosis, there were $7.7 \% \mathrm{NWT}$ to AFG and $7.7 \%$ NWT to CAS. Among the 20 bloodstream C. orthopsilosis, there were 5\% NWT to CAS, 5\% NWT to MFG, 15\% NWT to FLZ, 15\% NWT to VRZ, and $5 \%$ NWT to POZ.

Among the 480 non-bloodstream C. parapsilosis, there were 4.4\% SDD and $3.1 \% \mathrm{R}$ to FLZ, $1.3 \% \mathrm{I}$ and $1.5 \% \mathrm{R}$ to VRZ, $0.4 \% \mathrm{I}$ and $0.2 \%$ R to CAS, $1.5 \%$ I to AFG, $0.2 \%$ NWT to ITZ, $0.6 \%$ NWT to POZ, and $7.3 \%$ NWT to AMB. Among the 59 non-bloodstream C. metapsilosis, there were $10.2 \%$ NWT to AFG, $20.3 \%$ NWT to CAS, 3.4\% NWT to VRZ, and 1.7\% NWT to AMB. Among the 29 non-bloodstream C. orthopsilosis, there were 3.4\% NWT to CAS,
$3.4 \%$ NWT to MFG, $10.3 \%$ NWT to FLZ, $6.9 \%$ NWT to VRZ, and $3.4 \%$ NWT to POZ.

\section{MIC Values of the Nine Drugs Compared by Source (Non-bloodstream vs. Bloodstream)}

Although the general trend of the MIC ranges of the nine drugs for C. parapsilosis, C. metapsilosis, and C. orthopsilosis isolates were similar between bloodstream and non-bloodstream sources (Figure 1 and Supplementary Table 1), for some drugs, their MIC values differed between the two sources. The GM MICs of non-bloodstream C. parapsilosis for CAS, MFG, FLZ, VRZ, ITZ, and POZ were 0.555, 0.853, 0.816, 0.017, 0.076, and $0.042 \mathrm{mg} / \mathrm{L}$, respectively. These GM MICs were significantly higher than those of bloodstream C. parapsilosis, for which the GM MICs were 0.464, 0.745, 0.704, 0.015, 0.061, and $0.033 \mathrm{mg} / \mathrm{L}$, respectively, for CAS, MFG, FLZ, VRZ, ITZ, and POZ $(P<0.05)$. 


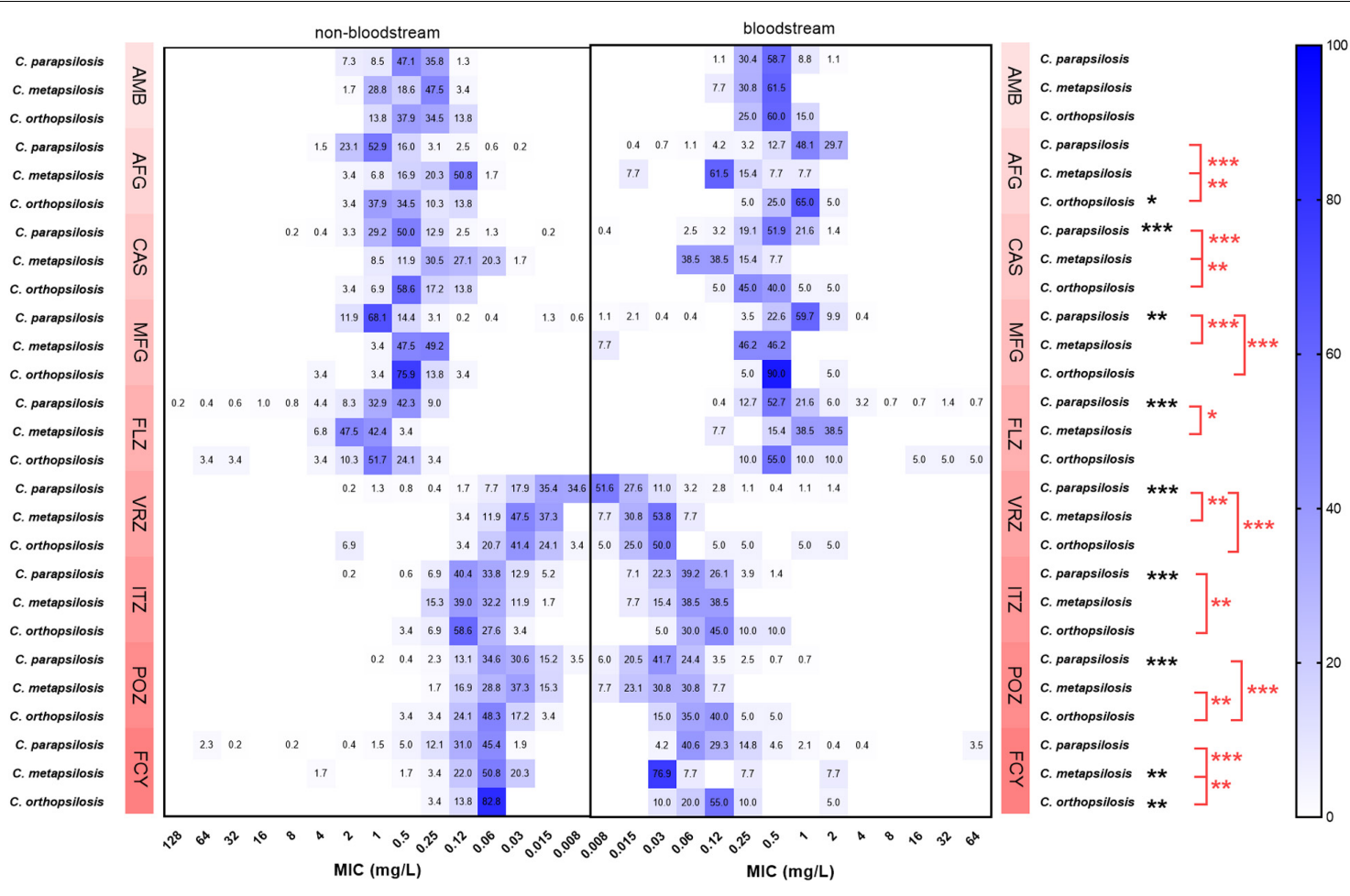

FIGURE 1 | Comparison of the proportions of minimum inhibitory concentration (MIC) distributions of $C$. parapsilosis complex isolates between bloodstream and non-bloodstream samples. Black *indicates $0.01<P<0.5$, statistically significant differences of MIC values between bloodstream and non-bloodstream isolates; black **indicates $0.001<P<0.01$, black ${ }^{* *}$ indicates $P<0.001$. Red *indicates $0.01<P<0.5$, statistically significant differences of MIC values between bloodstream C. parapsilosis complex species; red ${ }^{* \star}$ indicates $0.001<P<0.01$, red ${ }^{* * *}$ indicates $P<0.001$. FLZ, fluconazole; VRZ, voriconazole; ITZ, itraconazole; POZ, posaconazole; MFG, micafungin; AFG, anidulafungin; CAS, caspofungin; AMB, amphotericin B; FCY, 5-flucytosine.

The GM MIC of non-bloodstream C. metapsilosis for FCY $(0.071 \mathrm{mg} / \mathrm{L})$ was significantly higher than that of bloodstream isolates $(0.051 \mathrm{mg} / \mathrm{L})(P<0.05)$. In contrast, the GM MICs of non-bloodstream $C$. orthopsilosis for AFG and FCY were 0.522 and $0.069 \mathrm{mg} / \mathrm{L}$, respectively, which were significantly lower than those of bloodstream isolates, 0.812 and $0.113 \mathrm{mg} / \mathrm{L}$, respectively $(P<0.05)$.

\section{MIC Values of the Nine Drugs Compared Within Candida parapsilosis Complex Isolates}

The GM MIC of bloodstream C. parapsilosis for FLZ $(0.704 \mathrm{mg} / \mathrm{L})$ was significantly lower than that of C. metapsilosis $(0.997 \mathrm{mg} / \mathrm{L}) \quad(P<0.05$, Figure 1 and Supplementary Table 1). The GM MIC of bloodstream C. parapsilosis for MFG $(0.745 \mathrm{mg} / \mathrm{L})$ was significantly higher than those of C. metapsilosis $(0.264 \mathrm{mg} / \mathrm{L})$ and C. orthopsilosis $(0.518 \mathrm{mg} / \mathrm{L})$ $(P<0.05)$. The GM MIC of bloodstream C. parapsilosis for VRZ $(0.015 \mathrm{mg} / \mathrm{L})$ was significantly lower than those of $C$. metapsilosis $(0.023 \mathrm{mg} / \mathrm{L})$ and $C$. orthopsilosis $(0.041 \mathrm{mg} / \mathrm{L})(P<0.05)$. The GM MIC of bloodstream C. orthopsilosis for ITZ $(0.113 \mathrm{mg} / \mathrm{L})$ was significantly higher than that of C. parapsilosis $(0.061 \mathrm{mg} / \mathrm{L})$ $(P<0.05)$. The GM MIC of bloodstream C. orthopsilosis for POZ $(0.085 \mathrm{mg} / \mathrm{L})$ was significantly higher than those of C. parapsilosis $(0.033 \mathrm{mg} / \mathrm{L})$ and $C$. metapsilosis $(0.032 \mathrm{mg} / \mathrm{L})(P<0.05)$. The
GM MICs of bloodstream C. metapsilosis for CAS (0.115 mg/L), AFG $(0.150 \mathrm{mg} / \mathrm{L})$, and FCY $(0.051 \mathrm{mg} / \mathrm{L})$ were significantly lower than those of C. parapsilosis $(0.464,0.918$, and $0.136 \mathrm{mg} / \mathrm{L}$, respectively) and $C$. orthopsilosis $(0.378,0.812$, and $0.113 \mathrm{mg} / \mathrm{L}$, respectively) $(P<0.05)$.

\section{MIC Values of Bloodstream Candida parapsilosis Isolates With the Nine Drugs Compared Over Time}

The MIC distributions of C. parapsilosis isolates with the nine drugs were different between 2005-2018 (152 strains) and 20192020 (131 strains) (Figure 2). The GM MICs of strains collected from 2019 to 2020 for VRZ, POZ, and ITZ were 0.018, 0.040, and $0.073 \mathrm{mg} / \mathrm{L}$, respectively, which were significantly higher than those from 2005 to $2018(0.013,0.028$, and $0.052 \mathrm{mg} / \mathrm{L}$, respectively) $(P<0.05)$. In contrast, GM MICs of the strains collected from 2019 to 2020 for AMB, AFG, CAS, and FCY were $0.382,0.801,0.401$, and $0.096 \mathrm{mg} / \mathrm{L}$, respectively, which were significantly lower than those from 2005 to 2018 (0.430, 0.918, 0.464 , and $0.136 \mathrm{mg} / \mathrm{L}$, respectively) $(P<0.05)$.

\section{MIC Results for ISA}

A total of 143 C. parapsilosis complex isolates were tested for antifungal susceptibility to ISA (Table 3 ). The isolates all showed great susceptibility to the drug, with MIC ranges $\leq 0.008-1 \mathrm{mg} / \mathrm{L}$. 


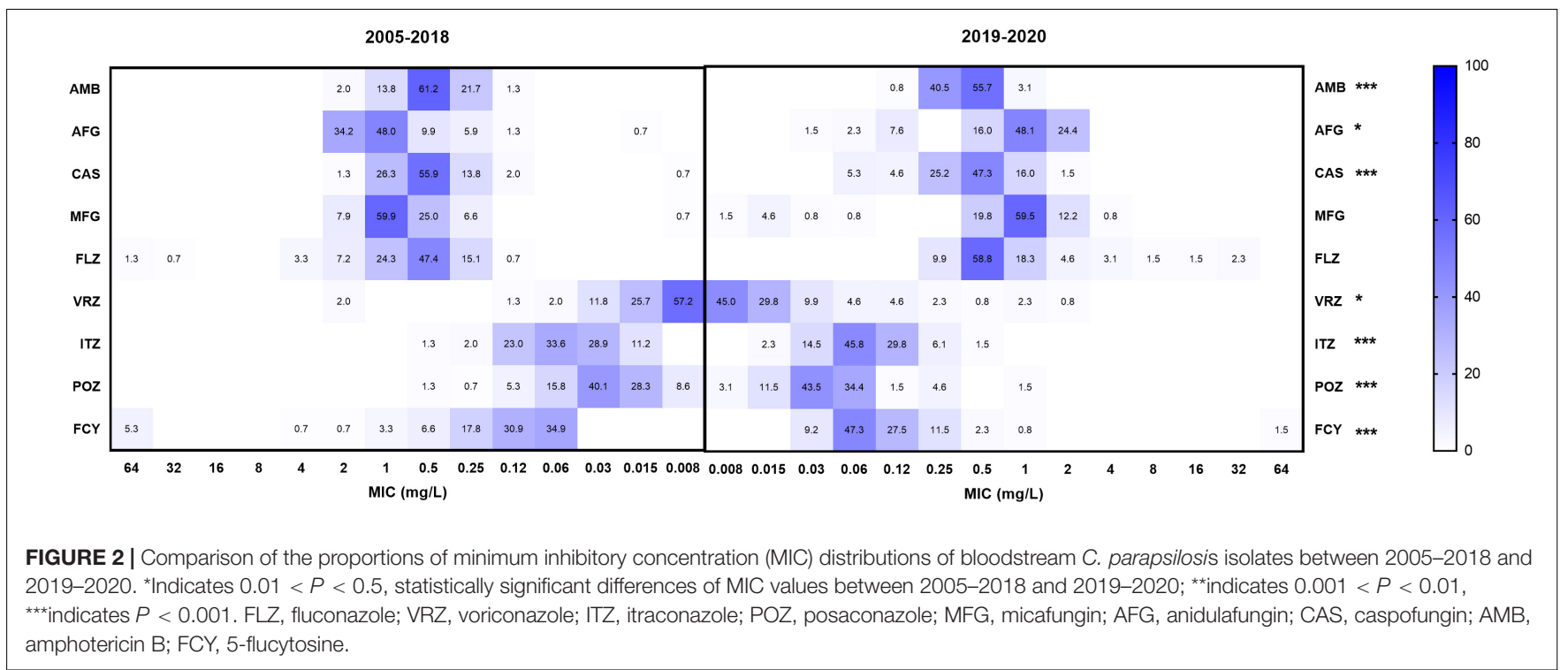

TABLE 3 | In vitro susceptibilities to isaconazole of the 143 C. parapsilosis complex isolates.

\begin{tabular}{|c|c|c|c|c|c|c|}
\hline \multirow[t]{2}{*}{ MIC terms (mg/L) } & \multicolumn{2}{|c|}{ C. parapsilosis } & \multicolumn{2}{|c|}{ C. metapsilosis } & \multicolumn{2}{|c|}{ C. orthopsilosis } \\
\hline & Blood $(n=61)$ & Non-blood $(n=56)$ & Blood $(n=10)$ & Non-blood $(n=12)$ & Blood $(n=2)$ & Non-blood $(n=2)$ \\
\hline MIC range & $\leq 0.008-1$ & $\leq 0.008-0.12$ & $\leq 0.008-0.03$ & $\leq 0.008-0.03$ & $\leq 0.008-0.06$ & $0.015-0.12$ \\
\hline MIC50 & $\leq 0.008$ & 0.015 & $\leq 0.008$ & $\leq 0.008$ & & \\
\hline MIC90 & 0.015 & 0.03 & 0.03 & 0.015 & & \\
\hline Modal MIC & $\leq 0.008$ & 0.015 & $\leq 0.008$ & $\leq 0.008$ & $\leq 0.008,0.06$ & $0.015,0.12$ \\
\hline GM MIC & 0.012 & 0.012 & 0.012 & 0.010 & 0.022 & 0.042 \\
\hline
\end{tabular}

MIC, minimum inhibitory concentration; MIC50, the MICs required to inhibit the growth of 50\% of the organisms; MIC90, the MICs required to inhibit the growth of $90 \%$ of the organisms; GM, geometric mean value.

\section{DISCUSSION}

Candida parapsilosis has become the second most prevalent non-albicans Candida species that causes invasive infections worldwide, ranging from $24.3 \%$ in Latin America to $12.9 \%$ in Asia-Pacific. From 2010 to 2019 in Beijing, the most frequently identified non-albicans Candida species were C. parapsilosis, C. tropicalis, and C. glabrata (Song et al., 2020). C. orthopsilosis and $C$. metapsilosis were reported to have much lower proportions ( $<1 \%$ of all Candida species) (Pfaller et al., 2019; Castanheira et al., 2020). Among the C. parapsilosis complexes that cause candidemia, C. orthopsilosis is responsible for approximately $40 \%$ in other countries, following C. parapsilosis with about $60 \%$, both of which are much higher than the results in this study (Maria et al., 2018; Vigezzi et al., 2019). The distribution of C. parapsilosis (89.6\%), C. metapsilosis (4.1\%), and C. orthopsilosis (6.3\%) from bloodstream (Table 2) is similar to what has been reported in Iran and Venezuela (Moreno et al., 2017; Arastehfar et al., 2019). Furthermore, this study also investigated the non-bloodstream C. parapsilosis complex, and there were nearly 1.8 times more of these isolates than bloodstream ones (Supplementary Table 1). The non-bloodstream sources included sputum, urine, stool, CVC, secretions, and the ear, by rank. Our data are consistent with the theory that $C$. parapsilosis complex species are a group of opportunistic invasive pathogenic fungi that often colonize the skin, mucous membranes, respiratory tract, intestinal tract, and reproductive tract, causing infections when the host's immunity is impaired. The species can also form biofilms on CVC and other medically implanted devices. The possibility of nosocomial crossinfections via the hands of the medical staff is alarming (Trofa et al., 2008; van Asbeck et al., 2009; Wang et al., 2016; Escribano et al., 2018; Tóth et al., 2019; Zhang et al., 2020).

The increasing number of nosocomial C. parapsilosis complex infections has raised concerns about conducting antifungal susceptibility tests to optimize clinical treatments (van Asbeck et al., 2008; Gonçalves et al., 2010; Cantón et al., 2011; Pemán et al., 2012; Ruiz et al., 2013; Gago et al., 2014). CLSI M60Ed2 updated the application rules for clinical breakpoints of C. parapsilosis complex: if a cryptic species of the complex is identified, the ECVs should be used instead of the breakpoints (CLSI, 2020). Therefore, this study categorized C. metapsilosis and C. orthopsilosis as WT and NWT based on the ECVs. Overall, the susceptible rates of bloodstream C. parapsilosis to AFG, CAS, MFG, FLZ, and VRZ ranged between 93.3 and 100\%, its WT rates to ITZ, POZ, and AMB were between 98.6 and $99.6 \%$, similar to the findings of other studies (da Silva et al., 2015; Moreno et al., 2017; Pfaller et al., 2019). The WT rates for 
bloodstream C. metapsilosis and C. orthopsilosis were between 92.3 and $100 \%$, while there were 15\% NWT for C. orthopsilosis for both FLZ and VRZ (Supplementary Table 2). Similarly, the data by the Shanghai Invasive Fungi Infection Group (IFIG) showed that the resistance rate of $C$. parapsilosis increased from 0 to approximately 5\% between 2017 and 2019 (data not published).

In this study, we also investigated the antifungal susceptibilities of non-bloodstream isolates. Notably, the MICs of non-bloodstream C. parapsilosis to CAS, MFG, FLZ, VRZ, ITZ, and POZ were significantly higher than those of bloodstream isolates $(P<0.05)$. This was also true for the MICs of $C$. metapsilosis to FCY $(P<0.05)$. However, MICs of nonbloodstream $C$. orthopsilosis to AFG and FCY were significantly lower than those of bloodstream isolates $(P<0.05$, Figure 1). Interestingly, the resistance rate of Klebsiella pneumoniae isolated from sputum also tends to be higher than those isolated from blood (Zhao-Yun et al., 2017; Feng et al., 2020). Because C. parapsilosis complex can transform from colonizing to invasive phenotypes (Escribano et al., 2018), the antifungal treatment and management of non-bloodstream species should be subject to strict supervision as for bloodstream species (Zhang et al., 2015; Pappas et al., 2018).

The supervision and management of antifungal treatments was performed by ECIFIG, which was launched in 2019 in Shanghai. Since launching, ECIFIG has been identifying organisms, testing their antifungal susceptibilities, and advocating the correct and rational use of antifungal drugs. It is believed that inappropriate drug exposure drives resistance. The mechanisms that cause drug resistance may naturally occur in less susceptible species and are then acquired in strains of susceptible organisms. Compared with MICs from 2005 to 2018, MIC values of bloodstream C. parapsilosis isolates collected from 2019 to 2020 decreased significantly for AMB, AFG, CAS, and FCY but increased significantly for VRZ, POZ, and ITZ $(P<0.05$, Figure 2$)$. This change indicated that attention should be paid to the clinical and agricultural use of azoles in Eastern China. It is also reflected in the opinion that an effective antifungal stewardship program is significant for controlling drug resistance.

The ECVs published in CLSI M59 are different among C. parapsilosis complex species for AMB, AFG, CAS, FLZ, ITZ, MFG, and VRZ. In this study, similar to the findings in CLSI M59, the MIC values of bloodstream C. metapsilosis were significantly lower than those of $C$. parapsilosis and C. orthopsilosis for AFG and CAS. MICs of C. parapsilosis were significantly higher than those of C. metapsilosis and C. orthopsilosis for MFG (Figure 1). However, MIC values of C. parapsilosis were significantly higher than those of C. metapsilosis for FLZ, while the ECV of C. parapsilosis for FLZ was lower than that of C. metapsilosis. Additionally, MICs of C. orthopsilosis were significantly higher than those of $C$. parapsilosis for ITZ and were also significantly higher than those of $C$. parapsilosis and C. metapsilosis for POZ. The ECVs for ITZ were equal for $C$. parapsilosis and C. orthopsilosis, and the ECVs for POZ were the same for all three species. This indicated that the MIC distributions of C. parapsilosis for FLZ and the distributions of C. orthopsilosis for ITZ and POZ might be higher than those in the CLSI
SENTRY program (Pfaller et al., 2011a,b, 2012, 2019; Pfaller, 2012; Castanheira et al., 2016). FLZ is recommended as firstline therapy for candidemia (Pappas et al., 2016), and clonal transmission of FLZ-resistant strains has been reported in Brazil and India (Govender et al., 2016; Thomaz et al., 2018; Singh et al., 2019). Therefore, the higher MIC distributions for azoles in this study is concerning. Apart from that, the MIC values for ISA in this study seemed quite low for C. parapsilosis complex isolates. This drug has shown potential as an alternative for candidemia treatment.

\section{CONCLUSION}

In summary, the higher MIC values of non-bloodstream isolates compared with bloodstream isolates should arouse attention for antifungal treatment and management of non-bloodstream isolates. Additionally, the increased MIC values for azoles of bloodstream C. parapsilosis isolates over the years and the higher MIC distributions for azoles in this study than in CLSI M59 raise concerns about the proper use of azoles in the clinic and environment. It may be worth establishing Eastern China's own ECV for C. parapsilosis complex to incorporate better clinical treatment and therapeutic drug monitoring. Finally, ISA has the potential to be an alternative treatment for candidemia.

\section{DATA AVAILABILITY STATEMENT}

The original contributions presented in the study are included in the article/Supplementary Material, further inquiries can be directed to the corresponding author/s.

\section{ETHICS STATEMENT}

All strains isolated from patients were preserved in the ECIFIG at Shanghai East Hospital. We conducted a retrospective study on the isolates and patient data, including age and gender from electronic laboratory records. The need for informed consent was waived by the Clinical Research Ethics Committee.

\section{AUTHOR CONTRIBUTIONS}

WW, LZ, and HX designed the experiments and supervised data analysis. JG, HS, DQ, and LW performed the antifungal susceptibility testing. JG and MZ wrote the manuscript. HX, LL, YL, CW, HD, HL, WZ, YWe, HZ, WX, YZ, SZ, RT, DW, and YWa collected and analyzed the data. All authors discussed the results and commented on the manuscript.

\section{FUNDING}

This study was supported by the National Natural Science Foundation of China (grant number 81971990), National Science and Technology Major Project (grant number 2018ZX10305409005-002), Shanghai Key Public Health Discipline (grant number 
GWV-10.1-XK04), Shanghai Excellent Technology Leader (grant number 20XD1434500), Clinical Laboratory Diagnostics (grant number PWZxk2017-09-1), and the Top-Level Clinical Discipline Project of Shanghai Pudong (grant number PWYgf 2018-05).

\section{REFERENCES}

Arastehfar, A., Khodavaisy, S., Daneshnia, F., Najafzadeh, M. J., Mahmoudi, S., Charsizadeh, A., et al. (2019). Molecular identification, genotypic diversity, antifungal susceptibility, and clinical outcomes of infections caused by clinically underrated yeasts, Candida orthopsilosis, and Candida metapsilosis: an iranian multicenter study (2014-2019). Front. Cell Infect. Microbiol. 9:264. doi: 10.3389/ fcimb.2019.00264

Asadzadeh, M., Ahmad, S., Al-Sweih, N., Hagen, F., Meis, J. F., and Khan, Z. (2019). High-resolution fingerprinting of Candida parapsilosis isolates suggests persistence and transmission of infections among neonatal intensive care unit patients in Kuwait. Sci. Rep. 9:1340.

Cantón, E., Pemán, J., Quindós, G., Eraso, E., Miranda-Zapico, I., Álvarez, M., et al. (2011). Prospective multicenter study of the epidemiology, molecular identification, and antifungal susceptibility of Candida parapsilosis, Candida orthopsilosis, and Candida metapsilosis isolated from patients with candidemia. Antimicrob. Agents Chemother. 55, 5590-5596. doi: 10.1128/aac.00466-11

Castanheira, M., Deshpande, L. M., Messer, S. A., Rhomberg, P. R., and Pfaller, M. A. (2020). Analysis of global antifungal surveillance results reveals predominance of Erg11 Y132F alteration among azole-resistant Candida parapsilosis and Candida tropicalis and country-specific isolate dissemination. Int. J. Antimicrob. Agents 55:105799. doi: 10.1016/j.ijantimicag.2019.09.003

Castanheira, M., Messer, S. A., Rhomberg, P. R., and Pfaller, M. A. (2016). Antifungal susceptibility patterns of a global collection of fungal isolates: results of the SENTRY Antifungal Surveillance Program (2013). Diagn. Microbiol. Infect Dis. 85, 200-204. doi: 10.1016/j.diagmicrobio.2016.02.009

Chow, J. K., Golan, Y., Ruthazer, R., Karchmer, A. W., Carmeli, Y., Lichtenberg, D., et al. (2008). Factors associated with candidemia caused by non-albicans Candida species versus Candida albicans in the intensive care unit. Clin. Infect. Dis. 46, 1206-1213.

CLSI (2020). Epidemiological Cutoff Values for Antifungal Susceptibility Testing, 3rd Edn. Wayne, PA: Clinical and Laboratory Standards Institute.

da Silva, B. V., Silva, L. B., De Oliveira, D. B., Da Silva, P. R., Ferreira-Paim, K., Andrade-Silva, L. E., et al. (2015). Species distribution, virulence factors, and antifungal susceptibility among Candida parapsilosis complex isolates recovered from clinical specimens. Mycopathologia 180, 333-343. doi: 10.1007/ s11046-015-9916-z

Escribano, P., Sánchez-Carrillo, C., Muñoz, P., Bouza, E., and Guinea, J. (2018). Reduction in percentage of Clusters of Candida albicans and Candida parapsilosis causing candidemia in a general hospital in Madrid, Spain. J. Clin. Microbiol. 56:e00574-18.

Feng, L., Su, B., Chang-Feng, Z., Shi-Jie, Z., and Xing-Xing, H. (2020). Clinical distribution characteristics and drug resistance analysis of Klebsiella pneumoniae. Chin. J. Clin. Pulmonol. 25, 1329-1332.

Gago, S., García-Rodas, R., Cuesta, I., Mellado, E., and Alastruey-Izquierdo, A. (2014). Candida parapsilosis, Candida orthopsilosis, and Candida metapsilosis virulence in the non-conventional host Galleria mellonella. Virulence 5, 278285. doi: $10.4161 /$ viru. 26973

García San Miguel, L., Pla, J., Cobo, J., Navarro, F., Sánchez-Sousa, A., Alvarez, M. E., et al. (2004). Morphotypic and genotypic characterization of sequential Candida parapsilosis isolates from an outbreak in a pediatric intensive care unit. Diagn. Microbiol. Infect. Dis. 49, 189-196. doi: 10.1016/j.diagmicrobio.2004. 03.017

Gil-Alonso, S., Quindós, G., Cantón, E., Eraso, E., and Jauregizar, N. (2019). Killing kinetics of anidulafungin, caspofungin and micafungin against Candida parapsilosis species complex: evaluation of the fungicidal activity. Rev. Iberoam. Micol. 36, 24-29. doi: 10.1016/j.riam.2018.12.001

Gonçalves, S. S., Amorim, C. S., Nucci, M., Padovan, A. C., Briones, M. R., Melo, A. S., et al. (2010). Prevalence rates and antifungal susceptibility profiles of the Candida parapsilosis species complex: results from a nationwide surveillance of

\section{SUPPLEMENTARY MATERIAL}

The Supplementary Material for this article can be found online at: https://www.frontiersin.org/articles/10.3389/fmicb. 2021.644000/full\#supplementary-material

candidaemia in Brazil. Clin. Microbiol. Infect. 16, 885-887. doi: 10.1111/j.14690691.2009.03020.x

Govender, N. P., Patel, J., Magobo, R. E., Naicker, S., Wadula, J., Whitelaw, A., et al. (2016). Emergence of azole-resistant Candida parapsilosis causing bloodstream infection: results from laboratory-based sentinel surveillance in South Africa. J. Antimicrob. Chemother. 71, 1994-2004. doi: 10.1093/jac/dkw091

Guinea, J. (2014). Global trends in the distribution of Candida species causing candidemia. Clin. Microbiol. Infect. 20(Suppl. 6), 5-10. doi: 10.1111/1469-0691. 12539

Guo, L. N., Xiao, M., Cao, B., Qu, F., Zhan, Y. L., Hu, Y. J., et al. (2017). Epidemiology and antifungal susceptibilities of yeast isolates causing invasive infections across urban Beijing. China. Future Microbiol. 12, 1075-1086. doi: 10.2217/fmb-2017-0036

Kuhn, D. M., Mikherjee, P. K., Clark, T. A., Pujol, C., Chandra, J., Hajjeh, R. A., et al. (2004). Candida parapsilosis characterization in an outbreak setting. Emerg. Infect. Dis. 10, 1074-1081.

Magobo, R. E., Naicker, S. D., Wadula, J., Nchabeleng, M., Coovadia, Y., Hoosen, A., et al. (2017). Detection of neonatal unit clusters of Candida parapsilosis fungaemia by microsatellite genotyping: results from laboratory-based sentinel surveillance, South Africa, 2009-2010. Mycoses 60, 320-327. doi: 10.1111/myc. 12596

Maria, S., Barnwal, G., Kumar, A., Mohan, K., Vinod, V., Varghese, A., et al. (2018). Species distribution and antifungal susceptibility among clinical isolates of Candida parapsilosis complex from India. Rev. Iberoam. Micol. 35, 147-150. doi: 10.1016/j.riam.2018.01.004

Modiri, M., Hashemi, S. J., Ghazvin, I. R., Khodavaisy, S., Ahmadi, A., Ghaffari, M., et al. (2019). Antifungal susceptibility pattern and biofilm-related genes expression in planktonic and biofilm cells of Candida parapsilosis species complex. Curr. Med. Mycol. 5, 35-42.

Moreno, X., Reviakina, V., Panizo, M. M., Ferrara, G., García, N., Alarcón, V., et al. (2017). Molecular identification and in vitro antifungal susceptibility of blood isolates of the Candida parapsilosis species complex in Venezuela. Rev. Iberoam. Micol. 34, 165-170.

Pappas, P. G., Kauffman, C. A., Andes, D. R., Clancy, C. J., Marr, K. A., OstroskyZeichner, L., et al. (2016). Clinical practice guideline for the management of Candidiasis: 2016 update by the infectious diseases society of America. Clin. Infect. Dis. 62, e1-e50.

Pappas, P. G., Lionakis, M. S., Arendrup, M. C., Ostrosky-Zeichner, L., and Kullberg, B. J. (2018). Invasive candidiasis. Nat. Rev. Dis. Primers 4:18026.

Pemán, J., Cantón, E., Quindós, G., Eraso, E., Alcoba, J., Guinea, J., et al. (2012). Epidemiology, species distribution and in vitro antifungal susceptibility of fungaemia in a Spanish multicentre prospective survey. J. Antimicrob. Chemother. 67, 1181-1187. doi: 10.1093/jac/dks019

Pfaller, M. A. (2012). Antifungal drug resistance: mechanisms, epidemiology, and consequences for treatment. Am. J. Med. 125, S3-S13.

Pfaller, M. A., Andes, D. R., Diekema, D. J., Horn, D. L., Reboli, A. C., Rotstein, C., et al. (2014). Epidemiology and outcomes of invasive candidiasis due to non-albicans species of Candida in 2,496 patients: data from the Prospective Antifungal Therapy (PATH) registry 2004-2008. PLoS One 9:e101510. doi: 10.1371/journal.pone.0101510

Pfaller, M. A., Diekema, D. J., Turnidge, J. D., Castanheira, M., and Jones, R. N. (2019). Twenty years of the SENTRY antifungal surveillance program: results for candida species from 1997-2016. Open Forum. Infect. Dis. 6, S79-S94.

Pfaller, M. A., Espinel-Ingroff, A., Canton, E., Castanheira, M., Cuenca-Estrella, M., Diekema, D. J., et al. (2012). Wild-type MIC distributions and epidemiological cutoff values for amphotericin B, flucytosine, and itraconazole and Candida spp. as determined by CLSI broth microdilution. J. Clin. Microbiol. 50, 2040-2046. doi: $10.1128 / \mathrm{jcm} .00248-12$

Pfaller, M. A., Messer, S. A., Moet, G. J., Jones, R. N., and Castanheira, M. (2011a). Candida bloodstream infections: comparison of species distribution 
and resistance to echinocandin and azole antifungal agents in intensive care unit (ICU) and non-ICU settings in the SENTRY antimicrobial surveillance program (2008-2009). Int. J. Antimicrob. Agents 38, 65-69. doi: 10.1016/j. ijantimicag.2011.02.016

Pfaller, M. A., Moet, G. J., Messer, S. A., Jones, R. N., and Castanheira, M. (2011b). Geographic variations in species distribution and echinocandin and azole antifungal resistance rates among Candida bloodstream infection isolates: report from the SENTRY antimicrobial surveillance program (2008 to 2009). J. Clin. Microbiol. 49, 396-399. doi: 10.1128/jcm.01398-10

Ruiz, L. S., Khouri, S., Hahn, R. C., Da Silva, E. G., De Oliveira, V. K., Gandra, R. F., et al. (2013). Candidemia by species of the Candida parapsilosis complex in children's hospital: prevalence, biofilm production and antifungal susceptibility. Mycopathologia 175, 231-239. doi: 10.1007/s11046-013-9616-5

Silva, S., Negri, M., Henriques, M., Oliveira, R., Williams, D. W., and Azeredo, J. (2012). Candida glabrata, Candida parapsilosis and Candida tropicalis: biology, epidemiology, pathogenicity and antifungal resistance. FEMS Microbiol. Rev. 36, 288-305. doi: 10.1111/j.1574-6976.2011.00278.x

Singh, A., Singh, P. K., De Groot, T., Kumar, A., Mathur, P., Tarai, B., et al. (2019). Emergence of clonal fluconazole-resistant Candida parapsilosis clinical isolates in a multicentre laboratory-based surveillance study in India. J. Antimicrob. Chemother. 74, 1260-1268. doi: 10.1093/jac/dkz029

Song, Y., Chen, X., Yan, Y., Wan, Z., Liu, W., and Li, R. (2020). Prevalence and antifungal susceptibility of pathogenic yeasts in China: a 10-year retrospective study in a teaching hospital. Front. Microbiol. 11:1401. doi: 10.3389/fmicb.2020. 01401

Strollo, S., Lionakis, M. S., Adjemian, J., Steiner, C. A., and Prevots, D. R. (2016). Epidemiology of hospitalizations associated with invasive Candidiasis, United States, 2002-2012(1). Emerg. Infect. Dis. 23, 7-13. doi: 10.3201/eid2301. 161198

Tavanti, A., Davidson, A. D., Gow, N. A., Maiden, M. C., and Odds, F. C. (2005). Candida orthopsilosis and Candida metapsilosis spp. nov. to replace Candida parapsilosis groups II and III. J. Clin. Microbiol. 43, 284-292. doi: 10.1128/jcm. 43.1.284-292.2005

Thomaz, D. Y., De Almeida, J. N. Jr., Lima, G. M. E., Nunes, M. O., Camargo, C. H., Grenfell, R. C., et al. (2018). An azole-resistant Candida parapsilosis outbreak: clonal persistence in the intensive care unit of a Brazilian teaching hospital. Front. Microbiol. 9:2997. doi: 10.3389/fmicb.2018.02997

Tóth, R., Nosek, J., Mora-Montes, H. M., Gabaldon, T., Bliss, J. M., Nosanchuk, J. D., et al. (2019). Candida parapsilosis: from genes to the bedside. Clin. Microbiol. Rev. 32:e0111-18.

Trofa, D., Gácser, A., and Nosanchuk, J. D. (2008). Candida parapsilosis, an emerging fungal pathogen. Clin. Microbiol. Rev. 21, 606-625. doi: 10.1128/cmr. 00013-08

van Asbeck, E., Clemons, K. V., Martinez, M., Tong, A. J., and Stevens, D. A. (2008). Significant differences in drug susceptibility among species in the Candida parapsilosis group. Diagn. Microbiol. Infect. Dis. 62, 106-109. doi: 10.1016/j. diagmicrobio.2008.04.019

van Asbeck, E. C., Clemons, K. V., and Stevens, D. A. (2009). Candida parapsilosis: a review of its epidemiology, pathogenesis, clinical aspects, typing and antimicrobial susceptibility. Crit. Rev. Microbiol. 35, 283-309. doi: 10.3109/ 10408410903213393
Vaz, C., Sampaio, P., Clemons, K. V., Huang, Y. C., Stevens, D. A., and Pais, C. (2011). Microsatellite multilocus genotyping clarifies the relationship of Candida parapsilosis strains involved in a neonatal intensive care unit outbreak. Diagn. Microbiol. Infect. Dis. 71, 159-162. doi: 10.1016/j.diagmicrobio.2011. 05.014

Vigezzi, C., Icely, P. A., Dudiuk, C., Rodríguez, E., Miró, M. S., Castillo, G. D. V., et al. (2019). Frequency, virulence factors and antifungal susceptibility of Candida parapsilosis species complex isolated from patients with candidemia in the central region of Argentina. J. Mycol. Med. 29, 285-291. doi: 10.1016/j. mycmed.2019.100907

Wang, H., Zhang, L., Kudinha, T., Kong, F., Ma, X. J., Chu, Y. Z., et al. (2016). Investigation of an unrecognized large-scale outbreak of Candida parapsilosis sensu stricto fungaemia in a tertiary-care hospital in China. Sci. Rep. 6:27099.

Xiao, M., Chen, S. C., Kong, F., Xu, X. L., Yan, L., Kong, H. S., et al. (2020). Distribution and antifungal susceptibility of Candida Species causing Candidemia in China: an update from the CHIF-NET study. J. Infect. Dis. 221, S139-S147.

Xiao, M., Fan, X., Chen, S. C., Wang, H., Sun, Z. Y., Liao, K., et al. (2015). Antifungal susceptibilities of Candida glabrata species complex, Candida krusei, Candida parapsilosis species complex and Candida tropicalis causing invasive candidiasis in China: 3 year national surveillance. J. Antimicrob. Chemother. 70, 802-810. doi: 10.1093/jac/dku460

Xiao, M., Sun, Z. Y., Kang, M., Guo, D. W., Liao, K., Chen, S. C., et al. (2018). Five-year national surveillance of invasive Candidiasis: species distribution and azole susceptibility from the China hospital invasive fungal surveillance net (CHIF-NET) study. J. Clin. Microbiol. 56:e00577-18.

Zhang, L., Xiao, M., Watts, M. R., Wang, H., Fan, X., Kong, F., et al. (2015). Development of fluconazole resistance in a series of Candida parapsilosis isolates from a persistent candidemia patient with prolonged antifungal therapy. BMC Infect. Dis. 15:340. doi: 10.1186/s12879-015-1086-6

Zhang, L., Yu, S.-Y., Chen, S. C.-A., Xiao, M., Kong, F., Wang, H., et al. (2020). Molecular characterization of Candida parapsilosis by microsatellite typing and emergence of clonal antifungal drug resistant strains in a multicenter surveillance in China. Front. Microbiol. 11:1320. doi: 10.3389/fmicb.2020. 01320

Zhao-Yun, X., Huai, Y., Yun, X., Jing, S., Zhong-Ling, Y., and Yang, H. (2017). Clinical distribution and drug resistance of Klebsiella pneumoniae in nonsputum samples. Chin. J. Hosp. Infect. 27, 1689-1692.

Conflict of Interest: The authors declare that the research was conducted in the absence of any commercial or financial relationships that could be construed as a potential conflict of interest.

Copyright (c) 2021 Guo, Zhang, Qiao, Shen, Wang, Wang, Li, Liu, Lu, Wang, Ding, Zhou, Zhou, Wei, Zhang, Xi, Zheng, Wang, Tang, Zeng, Xu and Wu. This is an open-access article distributed under the terms of the Creative Commons Attribution License (CC BY). The use, distribution or reproduction in other forums is permitted, provided the original author(s) and the copyright owner(s) are credited and that the original publication in this journal is cited, in accordance with accepted academic practice. No use, distribution or reproduction is permitted which does not comply with these terms. 